\title{
Suplementação nutricional para ovelhas em final de gestação: parâmetros nutricionais e metabólicos
}

\author{
Marco Túlio Santos Siqueira ${ }^{*}$; Thauane Ariel valadares de Jesus²; Adriana Lima Silva ${ }^{3}$; Maria Júlia Pereira de \\ Araújo $^{4}$; Luciano Fernandes Sousa ${ }^{5}$; Gilberto de Lima Macedo Júnior ${ }^{6}$
}

DOI: https://doi.org/10.35699/2447-6218.2020.24109

\begin{abstract}
Resumo
Objetivou-se avaliar o efeito da suplementação com dois níveis de concentrado na dieta de ovelhas no terço final da gestação sobre como afetam o consumo de matéria seca e metabólitos sanguíneos. Os experimentos foram realizados em 41 ovelhas com peso médio de $64,2 \mathrm{~kg}$. Os animais foram alojados em baias onde os tratamentos consistiam em dois níveis de concentrado como suplemento, distribuídos inteiramente ao acaso com medidas repetidas ao tempo (de acordo com a previsão de parto dos animais). A dieta foi composta por silagem de milho e concentrado na forma de suplemento, ofertada às 08:00 e 16:00 horas, sendo fornecidas ao animal de forma a obter 10\% de sobras do total ofertado de ração. As médias dos tratamentos foram avaliadas pelo teste de Tukey ao nível de significância de 5\%. Para as avaliações do período utilizou-se estudo de regressão ao nível de significância de 5\%. Dentre as variáveis analisadas, o consumo de matéria seca aumentou ao longo de todo o período experimental. Com relação aos metabólitos energéticos houve diferença apenas para o período analisado com aumento do LDL. Em relação às enzimas de atividade hepática, somente a aspartato amino transferase sofreu influência do período de avaliação. Sobre os metabólitos proteicos, ureia, creatinina e albumina sofreram efeito de redução ao longo do período experimental. A suplementação de ovelhas gestantes no terço final da gestação foi eficiente em melhorar o consumo no terço final da prenhez, sem causar danos metabólicos aos animais.
\end{abstract}

Palavras-chave: Condição corporal. Ganho de peso. Nutrição. Ovinos. Ruminante.

\section{Nutritional supplementation for ewes in late pregnancy: nutritional and metabolic parameters}

\begin{abstract}
The objective was to evaluate the effect of supplementation with two levels of concentrate in the diet of sheep in the final third of gestation on how they affect the consumption of dry matter and blood metabolites. The experiments were carried out on 41 sheep with an average weight of $64.2 \mathrm{~kg}$. The animals were housed in pens where the treatments consisted of two levels of concentrate as a supplement, distributed entirely at random with repeated measures over time (according to the prediction of birth of the animals). The diet consisted of corn silage and concentrated in the
\end{abstract}

${ }^{1}$ Universidade Federal de Uberlândia. Uberlândia, MG. Brasil.

https://orcid.org/0000-0002-2098-8568

${ }^{2}$ Universidade Federal de Uberlândia. Uberlandia, MG. Brasil.

https://orcid.org/0000-0003-3127-7697

${ }^{3}$ Universidade Federal de Uberlândia. Uberlandia, MG. Brasil.

https://orcid.org/0000-0002-9153-3317

${ }^{4}$ Universidade Federal de Uberlândia. Uberlandia, MG. Brasil.

https://orcid.org/0000-0002-7730-5828

${ }^{5}$ Universidade Federal de Tocantins. Araguaína, TO. Brasil.

https://orcid.org/0000-0002-6072-9237

${ }^{6}$ Universidade Federal de Uberlândia. Uberlandia, MG. Brasil.

https://orcid.org/0000-0001-5781-7917

*Autor para correspondência: marcotulio.s.siqueira@gmail.com

Recebido para publicação em 14 de Julho de 2020. Aceito para publicação 25 de Novembro de 2020

e-ISSN: 2447-6218 / ISSN: 2447-6218 / (C) 2009, Universidade Federal de Minas Gerais, Todos os direitos reservados. 
form of a supplement, offered at 08:00AM and 04:00PM hours, being provided to the animal in order to obtain 10\% of leftovers of the total offered of feed. The treatment means were evaluated by the Tukey test at a $5 \%$ significance level. For the evaluations of the period, a regression study was used at a significance level of $5 \%$. Among the variables analyzed, dry matter consumption increased throughout the experimental period. Regarding energy metabolites, there was a difference only for the period analyzed with an increase in LDL. Regarding liver enzymes, only aspartate amino transferase was influenced by the evaluation period. Regarding protein metabolites, urea, creatinine and albumin underwent a reduction effect throughout the experimental period. Supplementation of pregnant ewes in the final third of gestation was efficient in improving consumption in the final third of pregnancy, without causing metabolic damage to the animals.

Keywords: Body condition. Nutrition. Ruminant. Sheep. Weight gain.

\section{Introdução}

Alguns pontos positivos em fornecer suplementação com concentrado para ovelhas gestantes são frequentemente demonstrados, como aumento do peso ao nascer de cordeiros e aumento na produção de leite, o que afeta positivamente o peso à desmama e consequentemente $o$ peso ao abate, além de redução da duração do anestro pós-parto, tendo impacto direto no período de serviço e no intervalo de partos (Ortunho e Marçal, 2014).

Além dos maiores requerimentos nutricionais (maior demanda de energia e proteina na dieta) deve-se considerar dois aspectos que tendem a agravar a situação das ovelhas ao final da prenhez: primeiro, o aproveitamento da energia dos alimentos é muito reduzido (cerca de 5 a $22 \%$, comparando com valores de 40 a $60 \%$ para uma ovelha não gestante) decorrente do fato dos tecidos serem altamente especializados (Ortunho e Marçal, 2014) e, segundo, há perda do apetite das ovelhas pela redução do volume do trato gastrointestinal, devido ao maior espaço ocupado pelo feto e anexos gestacionais.

Diante disso, por meio da avaliação do perfil metabólico é possível saber se a nutrição está adequada e atende às exigências dos animais, uma vez que em algumas situações de desbalanço nutricional podem ocorrer alterações nas concentrações de alguns metabólitos sanguíneos, como ureia, proteínas totais e enzimas hepáticas (Kaneko et al., 2008).

Portanto, objetivou-se avaliar o consumo de matéria seca e metabólitos sanguíneos energéticos, enzimas hepáticas e metabólitos proteicos de ovelhas mestiças a partir de 135 dias de gestação até o momento do parto, suplementadas com duas quantidades de concentrado (300 e 400 gramas) na dieta.

\section{Material e métodos}

O experimento foi conduzido na Universidade Federal de Uberlândia, setor de pequenos ruminantes, em Uberlândia, Oeste de Minas Gerais, Brasil, durante os meses de julho e agosto de 2016 com duração de 33 dias. Foram utilizadas 41 ovelhas com idade superior a dois anos, gestantes, mestiça Santa x Inês Dorper, vacinadas (clostridioses, leptospirose, botulismo e raiva) e vermifugadas com Zolvix ${ }^{\circledR}$ (Novartis Saúde Animal, Basileia-Cidade, Basileia, França) na dose de 2,5 mg de Monepantel por kg de peso vivo.

Realizou-se ultrassonografia para diagnóstico de gestação, um mês após realização da cobertura, que ocorreu mediante sincronização de cio por meio de aplicação de $0,5 \mathrm{~mL}$ de hormônio luteolítico à base de Cloprostenol (Sincrocio ${ }^{\circledR}$ - Ouro Fino Saúde Animal, Cravinhos, São Paulo, Brasil) intramuscular, e exposição aos machos após 48 horas, permanecendo com os reprodutores por cinco dias. Após confirmação da prenhez, todas as matrizes gestantes foram mantidas em piquetes $\left(800 \mathrm{~m}^{2}\right)$, com dois animais por piquete, cultivados com pasto de capim Urochloa brizantha cultivar Marandu, adubados com $10 \mathrm{~kg}$ de ureia cada, recebendo suplemento proteico e energético na forma de proteinado, ajustado para consumo de $0,1 \%$ do peso vivo e com disponibilidade de água e sombra artificial até início do experimento. Os animais foram mantidos nos piquetes de março a julho de 2016 (lotação contínua), durante boa parte do período de estiagem da região, caracterizados por dias mais curtos, $285,3 \mathrm{~mm}$ de precipitação total durante os cinco meses e umidade relativa do ar média de 71,08\% segundo dados do CLiMA (Laboratório de climatologia e Meteorologia Ambiental), diminuindo assim a massa de forragem disponível e a qualidade da forrageira ofertada, causando restrição alimentar nos animais.

Para início do experimento esses animais foram confinados aos 135 dias de gestação com peso médio de $64,2 \mathrm{~kg}$ e escore de condição corporal (ECC) igual à 2 ,6. Foram mantidos até o momento do parto, em baias coletivas, equipadas com bebedouro, comedouro e saleiro e piso concretado com utilização de maravalha como cama para melhor conforto dos animais. As baias localizavam-se em galpão de alvenaria, com telhas de barro. As ovelhas foram distribuídas em dois tratamentos que se diferenciaram quanto ao nível de inclusão de concentrado na dieta, com 21 animais no tratamento com 300 gramas animal ${ }^{-1} \mathrm{dia}^{-1}$ e 20 animais no tratamento com 400 gramas animal $^{-1} \mathrm{dia}^{-1}$.

As ovelhas foram separadas de acordo com a previsão de parto, dessa forma o tratamento $300 \mathrm{~g}$ foi 
Suplementação nutricional para ovelhas em final de gestação: parâmetros nutricionais e metabólicos

utilizado em cinco baias, sendo as médias para este tratamento de 63,7kg e ECC 2,7. Enquanto o tratamento $400 \mathrm{~g}$ foi utilizado em outras cinco baias com médias para este tratamento de $64,7 \mathrm{~kg}$ e ECC 2,5 .

O experimento foi dividido em três fases de cinco dias cada, até o momento do parto, para avaliação do consumo de matéria seca (CMS). Os animais foram pesados no início do experimento e no dia do parto, para cálculo do consumo de alimento e obtenção do peso médio. A silagem de milho foi ofertada de modo a suprir o consumo de $2,5 \%$ do peso corporal, sendo dividida em duas refeições diárias, às 8:00 e 16:00 horas, pesadas logo antes do fornecimento em balança com precisão de cinco gramas. O concentrado utilizado na suplementação foi preparado em misturador vertical, em batidas de 200 $\mathrm{kg}$ e armazenado em barris até o momento da utilização. As porcentagens de cada ingrediente, bem como análise bromatológica da silagem são descritas na tabela 1.

Tabela 1 - Composição percentual do concentrado e análise bromatológica da silagem de milho

\begin{tabular}{|c|c|c|c|c|c|}
\hline Ingrediente & $\begin{array}{l}\text { Farelo de } \\
\text { milho }\end{array}$ & $\begin{array}{l}\text { Farelo de } \\
\text { soja }\end{array}$ & $\begin{array}{l}\text { Farelo de glúten de } \\
\text { milho/Promil }\end{array}$ & Sal mineral & Ureia protegida \\
\hline$\%$ & 60,00 & 22,00 & 15,00 & 2,25 & 0,75 \\
\hline $\begin{array}{l}\text { Silagem de } \\
\text { milho }\end{array}$ & $\begin{array}{l}\text { Matéria } \\
\text { seca }\end{array}$ & $\begin{array}{l}\text { Proteína } \\
\text { bruta }\end{array}$ & $\begin{array}{c}\text { Fibra em detergente } \\
\text { neutro }\end{array}$ & $\begin{array}{c}\text { Fibra em } \\
\text { detergente ácido }\end{array}$ & $\begin{array}{c}\text { Nutrientes } \\
\text { digestíveis totais* }\end{array}$ \\
\hline$\%$ & 30,86 & 7,89 & 40,00 & 23,45 & 68,39 \\
\hline
\end{tabular}

Todos os dias foram coletadas amostras de sobras de alimento de cada baia e após o final do período de coleta, estas amostras foram armazenadas em freezers horizontais a $-15^{\circ} \mathrm{C}$, para conservação dos nutrientes. As sobras foram mensuradas e sempre que os valores foram iguais à zero, aumentou-se a quantidade de silagem fornecida em $10 \%$ até atingir sobra equivalente a $10 \%$ do ofertado. Os teores de matéria seca foram obtidos pelo método INCT-CA G-003/1. Posteriormente foi realizado o cálculo de consumo de matéria seca através proposta por Maynard et al., 1984: (Eq. 1)

Sendo $\mathrm{CN}=$ consumo do nutriente $(\mathrm{kg})$; Cons = quantidade de alimento consumido $(\mathrm{kg}) ; \%$ cons $=$ teor do nutriente no alimento fornecido (\%); Sob = quantidade de sobra retirada $(\mathrm{kg}) ; \%$ sob $=$ teor do nutriente nas sobras (\%).

A determinação da concentração de glicose sanguínea foi realizada aos 135, 140 e 145 dias de gestação, sendo repetida no dia do parto. As colheitas foram realizadas por venopunção da jugular com auxílio de tubos Vacutainer $^{\circledR}$ (BD, São Paulo, São Paulo, Brasil) contendo fluoreto, realizadas no período da manhã, antes do fornecimento da primeira alimentação, com exceção do dia do parto, que foi realizada imediatamente após o mesmo, sem horário definido.

As coletas de sangue para avaliação dos componentes bioquímicos foram por venopunção jugular com auxílio de tubos Vacutainer ${ }^{\circledR}$ (BD, São Paulo, São Paulo, Brasil) sem anti-coagulante realizadas aos 135, 140, 145 e 150 dias de gestação, repetidas no momento do parto. Todas foram realizadas nos mesmos dias e horários das coletas para determinação da glicose. As amostras de sangue coletadas foram centrifugadas a $3500 \mathrm{rpm}$ por 10 minutos, os soros foram separados em alíquotas, guardados em microtubos e armazenados em freezer a $-5^{\circ} \mathrm{C}$ para posterior análise laboratorial. Os componentes bioquímicos utilizados para determinação do metabolismo energético foram: colesterol, triglicerídeos, HDL (lipoproteína de alta densidade), VLDL (lipoproteína de muito baixa densidade, calculado dividindo-se o valor de triglicerídeos por 5) e LDL (lipoproteína de baixa densidade, calculado através da fórmula proposta por Friedewald, Levv e Fredrickson (1972): LDL = colesterol total - HDL - VLDL); para determinação das enzimas hepáticas foram: aspartato aminotrasferase, gama glutamil tranferase e fosfatase alcalina; e para determinar metabolismo proteico foram: ácido úrico, ureia, creatinina, albumina e proteínas totais. Todas as amostras foram processadas em analisador bioquímico automatizado Bioplus ${ }^{\circledR} 2000$ (Bioplus Produtos para Laboratório Ltda., Barueri, São Paulo, Brasil), usando kit comercial da Lab Test Diagnóstica S. A. ${ }^{\circledR}$ (Lagoa Santa, Minas Gerais, Brasil).

Adotou-se o delineamento inteiramente casualizado com medidas repetidas ao tempo, sendo um tratamento com 21 repetições utilizando $300 \mathrm{~g}$ de concentrado e outro com 20 repetições utilizando $400 \mathrm{~g}$. Para avaliação do consumo de matéria seca utilizou-se as baias como repetição, sendo assim, para cada tratamento houve cinco baias como unidade de repetição. Todas as variáveis foram testadas quanto à normalidade (Shapiro-Wilk, 1965) e homocedasticidade (Levene, 1960) das variâncias do resíduo. Aceitos estes pressupostos os dados foram submetidos à análise de variância e as médias dos tratamentos comparadas pelo teste de Tukey ao nível de significância de 5\%. Para as avaliações do período utilizou-se estudo de regressão ao nível de significância de 5\%. 
Siqueira, M. T. S. et al.

\section{Resultados e discussão}

Não houve diferença estatística $(\mathrm{P}>0,05)$ para o consumo de matéria seca (CMS) entre os tratamentos, como demonstrado na tabela 2. O CMS foi de $1,850 \mathrm{~kg}$ dia $^{-1}$, o que corresponde a um consumo médio de 3,06\% do peso vivo (PV). O NRC (2007) determina que para ovelhas gestantes o CMS seja de 2,5 a 3,2 \% do PV. Portanto, observa-se que neste estudo o CMS esteve dentro do preconizado pelo comitê.

Tabela 2 - Consumo médio de matéria seca por baia e por animal ( $\left.\mathrm{kg} \mathrm{dia}^{-1}\right)$ no pré-parto em função dos tratamentos

\begin{tabular}{lccccc}
\hline Tratamento $(\mathbf{g})$ & $\mathbf{3 0 0}$ & $\mathbf{4 0 0}$ & MG & CV & P \\
\hline CMS/baia & 8,05 & 7,61 & 7,83 & 11,37 & 0,6551 \\
CMS/animal & 1,890 & 1,811 & 1,851 & 17,25 & 0,5182 \\
\hline
\end{tabular}

P: valor de 95\% de probabilidade (P<0,05); CV: coeficiente de variação (\%); MG: média geral.

No entanto, houve diferença ao longo do período experimental de modo que o CMS aumentou diariamente, obtendo valor máximo neste estudo de 2,066 kg de matéria seca ingerida por animal por dia (Figura 1). O mesmo ocorreu para o CMS da baia que se mostrou mais elevado no período de maior avanço gestacional das ovelhas. Tais valores mostram possivelmente a presença de consumo compensatório realizado pelos animais. Podendo ser uma explicação para o aumento no consumo durante todo o período experimental, visto que os animais eram mantidos a pasto, com baixa oferta de alimento e qualidade reduzida da forrageira (efeito climático relatado no material e método), o que possivelmente pode caracterizar um período de restrição alimentar na fase que antecedeu o experimento.

Figura 1 - Consumo de matéria seca por baia e por animal ao longo do período experimental

\section{Consumo de matéria seca ( $\mathrm{kg} / \mathrm{dia})$}

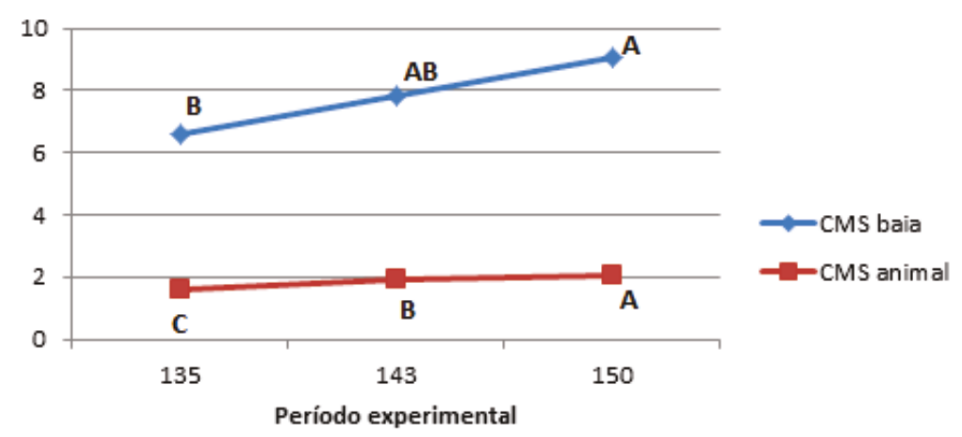

P baia: 0,0500; P animal: 0,0056; P: valor de 5\% de probabilidade.

Além disso, a suplementação pode ter estimulado o CMS destes animais, devido a sua palatabilidade e melhora do ambiente ruminal, uma vez que ela pode potencializar o crescimento microbiano o que por sua vez acaba elevando o CMS. Segundo o NRC (2007) este aumento de CMS após 130 dias de gestação é essencial para o adequado desenvolvimento fetal, visto que este período caracteriza-se pela fase de balanço energético negativo nessa espécie.
O CMS pode afetar positiva ou negativamente os metabólitos sanguíneos, visto que os mesmos são mensurados normalmente com o intuito de verificar se houve ou não falha nutricional nos animais a curto ou em longo prazo, além disso, a dieta é responsável em sua maioria pelo aporte energético e proteico do animal. Dessa forma, não houve efeito da suplementação sobre a glicose sanguínea entre os tratamentos (tabela 3).

Tabela 3 - Efeito da suplementação sobre a glicemia de ovelhas gestantes no pré-parto em função dos tratamentos

\begin{tabular}{lcccccc}
\hline Tratamento $(\mathbf{g})$ & $\mathbf{3 0 0}$ & $\mathbf{4 0 0}$ & MG & CV & P \\
\hline Glicemia (mg dL & (m) & 49,11 & 46,07 & 47,59 & 20,22 & 0,3632 \\
\hline
\end{tabular}

CV: coeficiente de variação (\%); MG: média geral; P: valor de 5\% de probabilidade.

A concentração média de glicose nos animais (47,6 $\mathrm{mg} \mathrm{dL}^{-1}$ ) ficou dentro do esperado na literatura que seria de 30,00 a $94,00 \mathrm{mg} \mathrm{dL}^{-1}$ segundo Silva et al. (2020). Com relação ao período experimental (Figura 2) não foram observadas grandes alterações entre os dias coletados. A fase final de gestação da ovelha apresenta elevada demanda deste nutriente em virtude do crescimento fetal. O nível de glicose tende a ser menor neste período, já que o feto demanda a mesma como maior fonte de energia, chegando a consumir $70 \%$ da produção 
Suplementação nutricional para ovelhas em final de gestação: parâmetros nutricionais e metabólicos

de glicose materna (Kaneko et al., 2008). Oliveira et

al. (2014), obtiveram valor médio de $44,29 \mathrm{mg} / \mathrm{dL}$ de glicose para ovelhas suplementadas ao final da gestação.

Figura 2 - Nível de glicose sanguínea ( $\mathrm{mg} \mathrm{dL}^{-1}$ ) em função do período experimental.

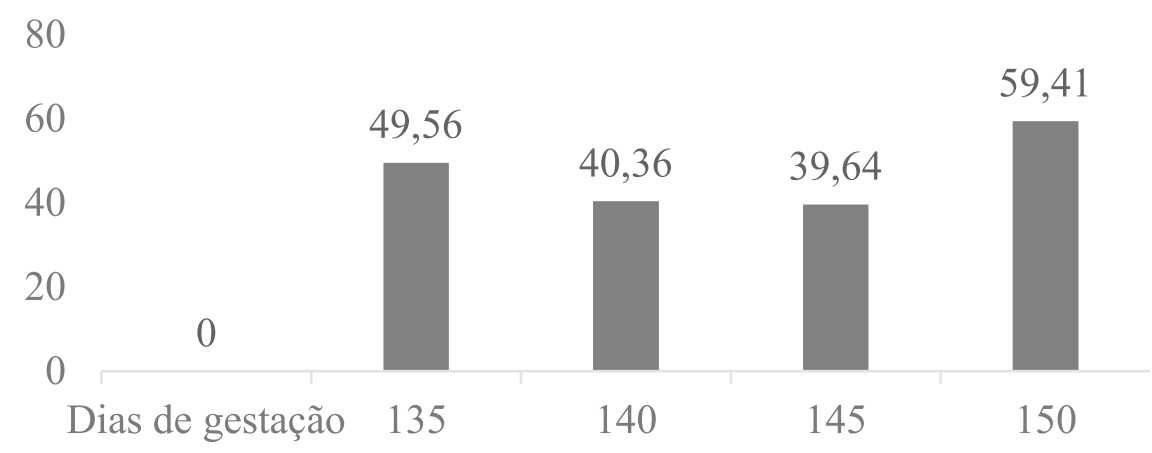

No presente trabalho, o valor de glicose atingiu o nível máximo $\left(59,41 \mathrm{mg} \mathrm{dL}^{-1}\right)$ aos 150 dias de prenhez (momento do parto), valor este dentro da faixa de referência descrita por Silva et al. (2020) (30,00 a 94,00 mg $\left.\mathrm{dL}^{-1}\right)$. Apesar dos valores não diferirem estatísticamente, demonstra bem quanto o metabolismo dos animais sofre severa modificação no momento do parto. Este quadro de hiperglicemia momentânea, que surge em decorrência do estresse desencadeado no momento do parto, é justificado pela elevação das concentrações sanguíneas do hormônio cortisol (Lima et al., 2016).

Além da glicose, existem outros metabólitos energéticos importantes que devem ser mensurados quando avaliamos algum tipo de dieta. Assim sendo, não foi observada diferença entre os tratamentos para as concentrações dos metabólitos energéticos, no entanto, para os metabólitos lipoproteína de baixa densidade (LDL), relação entre colesterol total e HDL e relação entre LDL e HDL (lipoproteína de alta densidade) observou-se diferença estatística dentro do período experimental como demonstrado na tabela 4.

Os valores de triglicerídeos encontraram-se de acordo com a referência, uma vez que a dieta dos animais foi constituída basicamente de silagem de milho, e alimentos volumosos são responsáveis pela maior produção de ácido acético no rúmen que é um dos ácidos graxos voláteis (AGV's) precursores da síntese de gordura em ruminantes. Em relação ao período nota-se queda dos níveis no momento do parto, o que está relacionado com o fato desses animais terem mobilizado reservas, uma vez que estiveram com glicemia (gráfico 2) sempre próximo ao limite inferior preconizado. A VLDL é responsável pelo transporte de triglicerídeos dos tecidos hepáticos para os tecidos periféricos, logo, é esperado que apresentassem comportamentos semelhantes (Santos et al., 2015). A mobilização de tecido adiposo, com liberação de ácidos

graxos livres, em ovelhas gestantes, pode ocorrer caso o suprimento energético seja inadequado, ou quando a concentração de glicose for reduzida para o crescimento fetal (Nasciutti, 2011). Esta variável permaneceu dentro da faixa de referência nos dois tratamentos e durante todo o período experimental, mas sempre próxima ao limite inferior.

Os teores de colesterol também estiveram dentro do esperado em todos os tratamentos e em quase todos os períodos (aumento numérico ao parto). Balikci et al. (2007) descreveram que ocorre aumento gradual do colesterol em ovelhas ao final da gestação, devido às concentrações de insulina, que atua diretamente do metabolismo do tecido adiposo durante o período gestacional, que acarreta no aumento das concentrações de colesterol e das lipoproteínas carreadoras deste, como a LDL, que apresentou efeito linear positivo durante o período experimental (tabela 4).

Os valores de HDL e LDL permaneceram dentro da faixa de referência nos dois tratamentos e durante todo o período experimental. O HDL é uma lipoproteína responsável pelo transporte de colesterol dos tecidos para o fígado para que este seja metabolizado, impedindo a deposição dele nas paredes das artérias (Kaneko et al, 2008). Em contrapartida, o LDL consiste de uma lipoproteína originária do fígado e responsável pelo transporte de colesterol deste para os tecidos periféricos. Quando há predominância de LDL, existe a tendência maior de deposição de colesterol nos tecidos, porque eles não conseguem catabolizar o excesso de LDL. A LDL teve efeito linear positivo ao longo do período, o que comprova a mobilização de reservas uma vez que os ácidos graxos presentes no fígado oriundos da mobilização precisam ser carreados para os tecidos para que não haja sobrecarga hepática. 
Siqueira, M. T. S. et al.

Tabela 4 - Concentração média dos metabólitos energéticos ( $\left.\mathrm{mg} \mathrm{dL}^{-1}\right)$ em função dos tratamentos e do período experimental em dias de gestação e no momento do parto

\begin{tabular}{|c|c|c|c|c|c|c|c|c|}
\hline \multirow{2}{*}{ Metabólitos } & \multicolumn{3}{|c|}{ Tratamento $(g)$} & \multirow{2}{*}{ MG } & \multirow{2}{*}{ CV } & \multirow{2}{*}{\multicolumn{2}{|c|}{ VR }} & \multirow{2}{*}{$\mathbf{P}$} \\
\hline & \multicolumn{2}{|c|}{300} & 400 & & & & & \\
\hline Colesterol $^{1}$ & \multicolumn{2}{|l|}{59,58} & 61,59 & 60,63 & 15,36 & \multicolumn{2}{|r|}{$14-126^{*}$} & 0,3696 \\
\hline Triglicerídeos ${ }^{1}$ & \multicolumn{2}{|l|}{20,94} & 23,98 & 22,53 & 13,14 & \multicolumn{2}{|r|}{$5-71^{*}$} & 0,2589 \\
\hline $\mathrm{HDL}^{1}$ & \multicolumn{2}{|l|}{24,62} & 24,70 & 24,66 & 11,41 & \multicolumn{2}{|r|}{$7-42^{* *}$} & 0,4782 \\
\hline $\mathrm{LDL}^{1}$ & \multicolumn{2}{|l|}{38,92} & 41,77 & 40,41 & 21,91 & \multicolumn{2}{|c|}{$4,3-95,5^{* *}$} & 0,1458 \\
\hline VLDL $^{2}$ & \multicolumn{2}{|l|}{4,18} & 4,79 & 4,50 & 20,48 & \multicolumn{2}{|r|}{$1-16,4^{*}$} & 0,4364 \\
\hline $\mathrm{CT} / \mathrm{HDL}^{1}$ & \multicolumn{2}{|l|}{2,66} & 2,97 & 2,82 & 34,68 & \multicolumn{2}{|r|}{-} & 0,2149 \\
\hline $\mathrm{LDL} / \mathrm{HDL}^{1}$ & \multicolumn{2}{|l|}{1,86} & 2,16 & 2,02 & 12,11 & \multicolumn{2}{|r|}{-} & 0,8893 \\
\hline \multirow{2}{*}{ Metabólitos } & \multicolumn{4}{|c|}{ Período (dias de gestação) } & \multirow{2}{*}{ VR } & \multirow{2}{*}{$\mathbf{P}$} & \multirow{2}{*}{$\mathbf{Y}$} & \multirow{2}{*}{$\mathbf{R}^{2}$} \\
\hline & 135 & 140 & 145 & Parto & & & & \\
\hline Colesterol $^{1}$ & 60,65 & 52,48 & 63,98 & 77,53 & $\begin{array}{l}14- \\
126^{*}\end{array}$ & 0,6325 & - & - \\
\hline Triglicerídeos ${ }^{1}$ & 18,92 & 22,68 & 26,25 & 23,46 & $5-71^{*}$ & 0,3510 & - & - \\
\hline $\mathrm{HDL}^{1}$ & 23,85 & 24,36 & 24,79 & 27,76 & $\begin{array}{l}10- \\
76,7^{*}\end{array}$ & 0,2498 & - & - \\
\hline $\mathrm{LDL}^{1}$ & 35,43 & 35,75 & 47,42 & 52,16 & $\begin{array}{l}1,2- \\
87^{*}\end{array}$ & 0,0152 & $\begin{array}{l}-133,631520 \\
+1,237363 x\end{array}$ & 89,86 \\
\hline $\mathrm{VLDL}^{2}$ & 3,78 & 4,53 & 5,25 & 4,69 & $\begin{array}{c}1- \\
16,4^{*}\end{array}$ & 0,3512 & - & - \\
\hline $\mathrm{CT} / \mathrm{HDL}^{1}$ & 2,77 & 2,64 & 2,94 & 3,29 & - & 0,0025 & $\begin{array}{c}-2,368236+ \\
0,037053 x\end{array}$ & 72,76 \\
\hline $\mathrm{LDL} / \mathrm{HDL}^{1}$ & 1,76 & 1,90 & 2,39 & 2,19 & - & 0,0071 & $\begin{array}{c}-3,041097+ \\
0,035829 x\end{array}$ & 65,71 \\
\hline
\end{tabular}

MG: média geral; CV: coeficiente de variação; *VR: valor de referência para ovinos segundo Silva et al. (2020); P: valor de 5\% de significância; HDL: lipoproteína de alta densidade; LDL: lipoproteína de baixa densidade; VLDL: lipoproteína de muito baixa densidade; CT/HDL: relação entre colesterol e lipoproteína de alta densidade; LDL/HDL: relação entre lipoproteína de baixa densidade e lipoproteína de alta densidade; ${ }^{1} m g d L^{-1}{ }^{2} g d L^{-1}$.

A relação CT/HDL está inversamente ligada à quantidade de HDL no sangue, ou seja, quanto mais baixa esta relação, maior a quantidade de HDL circulante e melhor é o carreamento de colesterol para o fígado, portanto, valores baixos de CT/HDL são desejáveis. Contudo, durante o período avaliado, houve aumento desses valores, indicando baixas taxas de HDL e consequentemente, maior deposição de colesterol nos tecidos. Da mesma forma, a relação LDL/HDL deve ser baixa, uma vez que valores elevados indicam maior concentração de LDL, que pode estar relacionado a um maior risco de doença nos animais pela deposição de colesterol e moléculas lipídicas nos tecidos (Kaneko et al., 2008). Houve efeito linear positivo para relação LDL/HDL durante o estudo, o que pode significar que o fígado está recebendo maiores quantidades de ácidos graxos que são convertidos em colesterol, triacilglicerol ou corpos cetônicos e, por isso tem uma necessidade maior de LDL para carrear essas moléculas para fora do seu tecido. Elevados valores das relações CT/HDL e LDL/HDL, associados à glicemia baixa ou limítrofe, indicam a mobilização de tecido adiposo de reserva para manutenção energética. Apesar de toda essa mobilização, não foi observado quadro clínico de toxemia da prenhez. Como não foi mensurado o beta-hidroxibutirato e ou ácidos graxos não esterificados não podemos inferir sobre possível toxemia da prenhez subclínica.

Na tabela 5 estão os valores da concentração das enzimas hepáticas em função dos tratamentos e do período experimental. Para a enzima aspartato aminotransferase observou-se diferença estatística dentro do período experimental. 
Suplementação nutricional para ovelhas em final de gestação: parâmetros nutricionais e metabólicos

Tabela 5 - Concentração média das enzimas hepáticas em função dos tratamentos e do período experimental em dias de gestação e no momento do parto

\begin{tabular}{|c|c|c|c|c|c|c|c|c|}
\hline \multirow{2}{*}{ Metabólitos } & \multicolumn{2}{|c|}{ Tratamento $(g)$} & \multirow{2}{*}{ MG } & \multirow{2}{*}{$\mathrm{CV}$} & \multirow{2}{*}{ VR } & \multirow{2}{*}{$\mathbf{P}$} & \multirow{2}{*}{$\mathrm{Y}$} & \multirow{2}{*}{$\mathbf{R}^{2}$} \\
\hline & 300 & 400 & & & & & & \\
\hline $\mathrm{AST}^{1}$ & 115,0 & 107,2 & 110,94 & 7,07 & $41-298$ & 0,210 & - & - \\
\hline $\mathrm{FA}^{1}$ & 87,81 & 89,89 & 88,90 & 10,87 & $49-826,9$ & 0,3625 & - & - \\
\hline $\mathrm{GGT}^{1}$ & 47,77 & 49,17 & 48,50 & 7,99 & $25-146$ & 0,4712 & - & - \\
\hline \multirow{2}{*}{ Metabólitos } & \multicolumn{4}{|c|}{ Período (dias de gestação) } & \multirow{2}{*}{ VR } & \multirow{2}{*}{$\mathbf{P}$} & \multirow{2}{*}{$\mathbf{Y}$} & \multirow{2}{*}{$\mathbf{R}^{2}$} \\
\hline & 135 & 140 & 145 & Parto & & & & \\
\hline $\mathrm{AST}^{1}$ & 138,7 & 117,1 & 80,29 & 85,96 & $41-298$ & 0,0074 & $\begin{array}{c}661,839403- \\
3,903828 \mathrm{x}\end{array}$ & 84,38 \\
\hline $\mathrm{FA}^{1}$ & 85,62 & 104,6 & 74,70 & 86,38 & $49-826,9$ & 0,8574 & - & - \\
\hline $\mathrm{GGT}^{1}$ & 45,70 & 45,02 & 52,85 & 56,76 & $25-146$ & 0,9234 & - & - \\
\hline
\end{tabular}

MG: média geral; CV: coeficiente de variação; VR: valor de referência para ovinos segundo Silva et al. (2020); P: valor de 5\% de significância; AST: aspartato aminotransferase; FA: fosfatase alcalina; GGT: gama glutamil transferase; ${ }^{1} U L^{-1}$.

A aspartato aminotransferase (AST) é uma enzima citoplasmática e mitocondrial, presente em vários tecidos como fígado, músculos esquelético e cardíaco. Observou-se efeito linear negativo para esta enzima quando se avaliou o período experimental, no presente estudo, as concentrações de AST estiveram dentro do intervalo considerado normal para a espécie (Silva et al., 2020), o que indica que esses animais não desenvolveram lesão hepática.

Já a fosfatase alcalina é uma enzima sintetizada em vários tecidos, sendo as maiores concentrações no intestino, rins, ossos e fígado (Kaneko et al., 2008). Aumento nos níveis de fosfatase alcalina no plasma sanguíneo indicam diversas condições patológicas, como a sobrecarga hepática. De forma geral, os valores desta enzima estiveram dentro da faixa de referência (Silva et al., 2020), também indicando bom funcionamento hepático pelos animais.

A GGT deve ser levada em consideração juntamente com a AST, já que ambas podem indicar se há ou não ocorrência de injúrias ao tecido hepático. Os níveis de GGT podem apresentar aumento imediato caso haja lesão hepática aguda, pois pode ocorrer liberação de fragmentos da membrana que contenham a enzima (Paula, 2015). Em ambos os tratamentos essa enzima está dentro dos valores de referência descritos por Silva et al. (2020), indicando que o fígado desses animais encontravam-se em um metabolismo adequado.

Apesar da mobilização de tecido gorduroso comprovada pelos valores da LDL e das relações CT/HDL e LDL/HDL não ocorreu sobrecarga hepática como demonstra as médias dos valores das enzimas hepáticas. Pode-se observar que todos os metabólitos energéticos e enzimas hepáticas ficaram dentro dos valores indicados como ideais na literatura. Tal fato pode estar relacionado com a suplementação e aumento do CMS ao longo do período experimental proporcionando menores chances de casos de toxemia da prenhez, que foi o caso deste estudo, onde nenhum animal apresentou a sintomatologia clínica.

Os metabólitos energéticos são eficientes para avaliar o status energético do animal e da dieta utilizada, no entanto para obtenção de resultados mais complexos faz-se necessária a avaliação dos metabólitos proteicos, onde em conjunto, são utilizados para avaliar o animal ou dieta em questão. Não houve efeito dos tratamentos sobre os metabólitos proteicos ao longo do experimento, no entanto houve efeito da utilização da suplementação com concentrado em diferentes níveis durante o período, sobre a concentração dos metabólitos proteicos ureia, creatinina e albumina (tabela 6).

O ácido úrico representa de forma indireta o crescimento de microrganismos no rúmen, uma vez que é utilizado pelos mesmos após a transformação em amônia como fator de crescimento microbiano, sendo utilizado para a síntese de proteína microbiana, tornando-se assim disponível para o ruminante (Paula, 2015). Sendo assim, foi possível observar que os valores se mostraram equilibrados ao longo do período experimental, e apesar de estar próximo ao limite inferior, não apresentou diferença estatística.

Não houve diferença estatística entre os tratamentos para proteínas totais, que manteve seus valores dentro do esperado (Silva et al., 2020). Segundo Kaneko et al. (2008), os níveis de proteínas totais tendem a apresentar queda nos períodos médio e final da gestação, devido à maior necessidade fisiológica que o crescimento do feto e o desenvolvimento do úbere impõem. No presente estudo observa-se uma queda numérica sem efeito estatístico. 
Siqueira, M. T. S. et al.

Observou-se queda para as variáveis creatinina e ureia. A ocorrência de redução desses metabólitos proteicos com o avanço da gestação pode estar associada à restrição alimentar que estes animais sofreram a pasto antes do experimento, uma vez que esses metabólitos respondem rápido ao efeito da ração.

Tabela 6 - Concentração média dos metabólitos proteicos em função dos tratamentos e do período experimental em dias de gestação e no momento do parto (150 dias)

\begin{tabular}{|c|c|c|c|c|c|c|c|c|}
\hline \multirow{2}{*}{ Metabólitos } & \multicolumn{2}{|c|}{ Tratamento $(g)$} & \multirow{2}{*}{ MG } & \multirow{2}{*}{$\mathrm{CV}$} & \multirow{2}{*}{ VR } & \multirow{2}{*}{$\mathbf{P}$} & \multirow{2}{*}{$\mathbf{Y}$} & \multirow{2}{*}{$\mathbf{R}^{2}$} \\
\hline & 300 & 400 & & & & & & \\
\hline Ácido úrico ${ }^{1}$ & 0,36 & 0,41 & 0,38 & 42,81 & $0-1,7$ & 0,4853 & - & - \\
\hline Ureia $^{1}$ & 23,59 & 21,31 & 22,39 & 11,24 & $10-92$ & 0,2547 & - & - \\
\hline Creatinina $^{1}$ & 0,99 & 0,99 & 0,99 & 13,25 & $1,2-1,9$ & 0,6541 & - & - \\
\hline Albumina $^{2}$ & 3,11 & 3,41 & 3,27 & 8,74 & $1,1-5,2$ & 0,2014 & - & - \\
\hline Proteínas totais ${ }^{2}$ & 5,64 & 5,75 & 5,70 & 7,69 & $3,1-10,7$ & 0,8896 & - & - \\
\hline \multirow{2}{*}{ Metabólitos } & \multicolumn{4}{|c|}{ Período (dias de gestação) } & \multirow{2}{*}{ VR } & \multirow{2}{*}{$\mathbf{P}$} & \multirow{2}{*}{$\mathbf{Y}$} & \multirow{2}{*}{$\mathbf{R}^{2}$} \\
\hline & 135 & 140 & 145 & Parto & & & & \\
\hline Ácido úrico ${ }^{1}$ & 0,36 & 0,43 & 0,35 & 0,38 & $0-1,7$ & 0,1025 & - & - \\
\hline Ureia $^{1}$ & 27,90 & 19,46 & 20,29 & 20,23 & $10-92$ & 0,0487 & $\begin{array}{c}85,176495- \\
0,443540 x\end{array}$ & 52,02 \\
\hline Creatinina $^{1}$ & 1,13 & 0,96 & 0,97 & 0,72 & $1,2-1,9$ & 0,0020 & $\begin{array}{l}4,404662- \\
0,024255 x\end{array}$ & 85,90 \\
\hline Albumina $^{2}$ & 3,94 & 2,81 & 3,16 & 2,95 & $1,1-5,2$ & 0,0112 & $\begin{array}{c}10,746209- \\
0,052822 x\end{array}$ & 45,65 \\
\hline Proteínas totais ${ }^{2}$ & 6,08 & 5,48 & 5,73 & 5,13 & $3,1-10,7$ & 0,8742 & - & - \\
\hline
\end{tabular}

MG: média geral; CV: coeficiente de variação; VR: valor de referência para ovinos segundo Silva et al. (2020); P: valor de $5 \%$ de significância; ${ }^{1}$ mg $d L^{-1} ;{ }^{2} g d L^{-1}$.

A ureia apresentou diferença estatística ao longo do período experimental, apresentando resposta linear negativa, apesar de todos os valores se encontrarem dentro dos valores de referência preconizados. Para o período 135 dias observa-se maior concentração de ureia em relação aos outros períodos que tendem a diminuir conforme se aumenta o tempo de gestação. Parte da proteína que chega ao rúmen é transformada em amônia, para ser utilizada pela microbiota ruminal na produção de proteína microbiana. Quando há falta de carboidratos na dieta a amônia é absorvida pela parede ruminal e levada ao fígado sendo transformada em ureia. Esta pode ser eliminada na urina ou no leite em caso de animal em lactação (Rennó et al., 2008). A concentração sanguínea de ureia possui relação direta com o aporte proteico da ração, perfil de aminoácidos e qualidade da proteína ingerida, podendo então indicar o excesso ou a deficiência do nutriente na dieta (Kaneko et al., 2008). Deste modo, é possível que tenha havido melhora do sinergismo com o concentrado fornecido, visto que antes do experimento estes animais se alimentavam de pasto de baixa qualidade e proteinado.

Os valores de creatinina ficaram abaixo da faixa normal de acordo com Silva et al. (2020) e não houve diferença estatística entre tratamentos, já no período houve resposta linear negativa, o que pode ter sido causado devido ao fato dos animais estarem confinados, o que resulta em baixo consumo de energia pelo músculo, uma vez que a creatinina tem estreita relação com a massa muscular que varia de acordo com grau de exercício realizado pelos animais.

Houve influência do período sobre o parâmetro albumina, de modo que os valores diminuíram estatisticamente conforme se aumentou o período gestacional, demonstrando resposta linear negativa. A albumina é uma proteína transportadora especialmente de lipídeos, que no fim da gestação foram altamente mobilizados. Comparando com os dados de Silva et al. (2020), esta variável fica dentro dos valores propostos pelo autor, visto que os animais utilizados no estudo se assemelham geneticamente e são, em sua maioria, endêmicos da mesma região. Portanto, presume-se que não houve falha proteica na dieta, uma vez que os animais não tiveram divergências no metabolismo proteico em comparação com a literatura, de modo que a suplementação conseguiu corrigir a deficiência proteica dos animais causada na época que antecedeu o período experimental. 
Suplementação nutricional para ovelhas em final de gestação: parâmetros nutricionais e metabólicos

\section{Conclusão}

A suplementação de ovelhas no terço final da gestação com diferentes níveis de concentrado, exerce influência sobre o consumo de matéria seca, aumentando o mesmo, e estabilizando a mobilização de reservas corporais, sem causar distúrbios metabólicos importantes aos animais.

\section{Aprovação do Comitê de Ética}

O protocolo experimental deste trabalho foi aprovado pela Comissão de Ética na Utilização de Animais (CEUA) da Universidade Federal de Uberlândia sob o número 016/16.

\section{Referências}

Balikci, E.; Yildiz, A.; Gürdogan, F. 2007. Blood metabolite concentrations during pregnancy and postpartum in Akkaraman ewes. Small Ruminant Research, 67: 247-251.

Friedewald, W. T.; Levv, R. I.; Fredrickson, D. S. 1972. Estimation of the concentration of low-density lipoprotein in plasma, without use of the preparative ultracentrifuge. Clin Chem, 18: 499-502.

Kaneko, J. J., Harvey, J. W., Bruss, M. L. (Eds.) 2008. Clinical Biochemistry of Domestic Animals. San Diego: Academic Press.

Levene, H. 1960. Robust Test for Equality of Variances, in I. O. et al., ed., 'Contributions to Probability and Statistics: Essays in Honor of Harold Hotteling', Stanford University Press, California, United States, 278-292.

Lima, E. H. F; Mendonça, C. L.; Cajueiro, J. F. P.; Carvalho, C. C. D.; Soares, P. C.; Souto, R. J. C.; Drummond, A. R. F; Afonso, J. A. B. 2016. Efeito da 75 monensina sódica sobre o perfil metabólico de ovelhas antes e após o parto. Ciência Animal Brasileira, 17: 105-118.

Maynard, L. A.; Loosli, J. K.; Hintz, H. F.; Warner, R. G. 1984. Nutrição animal. 3. ed. Rio de Janeiro: F. Bastos.

Nasciutti, N. R. 2011. Perfil metabólico em ovelhas santa Inês com baixo escore de condição corporal no periparto. Uberlândia: Universidade Federal de Uberlândia, 41 f. Dissertação mestrado. Disponível em: https://repositorio.ufu.br/handle/123456789/13007.

Nacional Research Council - NRC. 2007. Nutrient requirements of small ruminants: Sheep, goats, cervids, and new world camelids. Washington, D. C.: National Academy Press.

Oliveira, R. P. M.; Maduro, A. H. P.; Lima, E. S.; Oliveira, F. F. 2014 Perfil metabólico de ovelhas Santa Inês em diferentes fases de gestação criadas em sistema semi-intensivo no Estado do Amazonas. Ciência Animal Brasileira, v.15: 81-86.
Ortunho, V. V.; Marçal, W. S. 2014. Perfil proteico de fêmeas ovinas suplementadas com minerais. Revista Brasileira de Higiene e Sanidade Animal, 8: 189-205.

Paula, C. G. 2015. Suplementação com melaço de soja na dieta de ovinos: parâmetros sanguíneos, consumo, digestibilidade e comportamento ingestivo. Uberlândia: Universidade Federal de Uberlândia, 41 f. Dissertação mestrado. Disponível em: https://repositorio.ufu.br/ bitstream/123456789/13154/1/SuplementacaoMelacoSoja.pdf.

Renoó, L. N.; Valadares Filho, S. de C.; Paulino. M. F.; Leão, M. I.; Valadares. R. F. D.; Rennó. F. P.; Paixão, M. L. 2008. Níveis de uréia na ração de novilhos de quatro grupos genéticos: parâmetros ruminais, uréia plasmática e excreções de uréia e creatinina. Revista Brasileira de Zootecnia, 38: 556-562.

Rodrigues, R. C. 2010. Métodos de análises bromatológicas de alimentos: métodos físicos, químicos e bromatológicos. Pelotas.

Santos, R. P; Sousa, L. F.; Sousa, J. T. L.; Andrade, M. E. B.; Macedo Júnior, G. L.; Silva, S. P. 2015. Parâmetros sanguíneos de cordeiros em crescimento filhos de ovelhas suplementadas com níveis crescentes de propilenoglicol. Revista Brasileira de Ciências Agrárias, 10: 473-478.

Shapiro, S. S.; Wilk, M. B. 1965. An Analysis of Variance Test for Normality, (Complete Samples). Biometrika, 52: 591-611. http:// dx.doi.org/10.2307/2333709.

Silva, D. A. P; Varanis, L. F. M.; Oliveira, K. A.; Sousa, L. M.; Siqueira, M. T. S.; Macedo Júnior, G. L. 2020. Parâmetros de metabólitos bioquímicos em ovinos criados no Brasil. Caderno de Ciências Agrárias, 12, 01-08. DOI: https://doi.org/10.35699/2447-6218.2020.20404. 